\title{
La sournoise émergence des cités dites sécurisées en Turquie \\ Le cas de l'arrondissement de Beykoz (Istanbul)
}

\section{Jean-François Pérouse, Istanbul}

\section{Introduction}

Bien qu'il s'agisse en Turquie d'un phénomène saillant depuis une dizaine d'années, la réflexion turque en sciences sociales sur les cités sécurisées, demeure assez limitée (BALI 1999; ÖNCÜ 1999; Bartu 2002). Les hésitations et flottements du vocabulaire sont à cet égard révélateurs, comme si cette réalité socio-spatiale n'était pas encore vraiment formulée ni assumée: on parle indifféremment d'îlot fortifié (müstahkem adacik), de cité fermée (kapali site), cité protégée (korunakli site) ou de cité sécurisée (güvenlikli site). ÖNCÜ (1999), dans son article pionnier, caractérise ces cités comme une expression à la fois de la fuite des centres par les classes moyennes et supérieures et de la volonté de ces classes de participer à la culture de consommation globale. Si son approche a le mérite de recadrer l'apparition des cités sécurisées dans un large contexte - ouverture de l'économie turque et diffusion du «rêve de la maison individuelle»-, elle tend à négliger les mécanismes concrets de l'offre et de la construction dans un segment de marché restreint correspondant à une clientèle à très hauts revenus. BARTU (2002), quant à elle, s'intéresse à une seule gated community du nord-ouest d'Istanbul, de grande taille il est vrai, et plus spécifiquement au discours communautariste (du traditional neighborhood, créé de toutes pièces), importé des Etats-Unis, qui y est développé comme argument publicitaire.

Aussi, le recours aux travaux relatifs aux processus ségrégatifs en Amérique Latine (АмAтo 1970; Fischer \& Parnreiter 2002), malgré l'antériorité du phénomène dans les pays concernés, apparaît donc indispensable à un meilleur cadrage de l'analyse, de même que le recours aux études menées de longue date aux Etats-Unis (Davis 1990; Blakely \& SNyder 1997). Compte tenu du caractère récent et encore peu étudié du phénomène en Turquie, notre ambition sera réduite. Elle visera, à l'instar de ce qu'ont proposé Coy et PöHLER (2002) pour le Brésil, à réfléchir, à travers un exemple sciemment circonscrit, au processus d'émergence et de différenciation de ces cités, dans un contexte précis. En ce sens, il s'agira de poser les bases pour une recherche plus systématique qui suppose, selon nous, un certain acquis monographique préalable. L'objet de cet article, focalisé à dessein sur un ter- ritoire limité au sein de l'immensité stambouliote, est de proposer des critères d'analyse et de différenciation de ces cités, dans un contexte donné, afin de prendre la mesure du phénomène dans sa phase d'émergence.

Beykoz est l'un des 32 arrondissements constitutifs du département d'Istanbul (fig.1), un des plus étendus en l'occurrence (soit $427 \mathrm{~km}^{2}$ ). Sa caractéristique principale est, en raison d'une situation relativement périphérique, au nord-est de l'aire urbaine - l'arrondissement est délimité, à l'ouest, par le Bosphore et, au nord, par la mer Noire -, de compter encore de nombreux terrains non urbanisés, couverts par la forêt. Les vastes terrains périphériques (mücavir alanlari) de Beykoz - qui représentent plus des $2 / 3$ de la superficie totale de l'arrondissement et échappent à la gestion de la municipalité élue -, en situation de quasi non droit sur le plan de la planification urbaine, apparaissent dès lors comme les zones de prédilection des constructeurs de cités. Beykoz fait aussi partie des arrondissements ayant enregistré une très forte croissance annuelle ces dernières années (plus de 3\% en moyenne, entre 1990 et 2000), alors même que l'essentiel de l'arrondissement n'a pas encore été officiellement ouvert à l'urbanisation: on est en fait dans un régime d'autorisation provisoire de construction, qui s'éternise et couvre tous les abus; ce qui peut surprendre pour un arrondissement de plus de 180000 habitants.

En outre, du fait des aménités qu'il offre - dans le système dominant des valeurs environnementales -, cet arrondissement apparaît comme l'un des plus convoités par les fabricants de cités sécurisées et les classes visées par ceux-ci. En effet, il combine la proximité du Bosphore, et les perspectives sur celui-ci, la proximité de la mer Noire et celle des forêts qui coupent encore partiellement la mer Noire de l'aire urbaine qui s'étend au sud. De plus, et ce surtout depuis le traumatisme des tremblements de terre de 1999, le sous-sol y est réputé solide, du point de vue des risques sismiques. Dans ce contexte, il n'est pas surprenant de constater que Beykoz compte parmi les arrondissements qui concentrent le plus de cités sécurisées (expression que nous considérerons comme équivalente de l'anglais gated communities). Surtout, on peut comprendre que ce soit à Beykoz qu'aient apparu les premières cités sécurisées d'Istanbul (et probablement de Turquie). Près de 30 ont été repérées et identifiées en décembre 2002. 


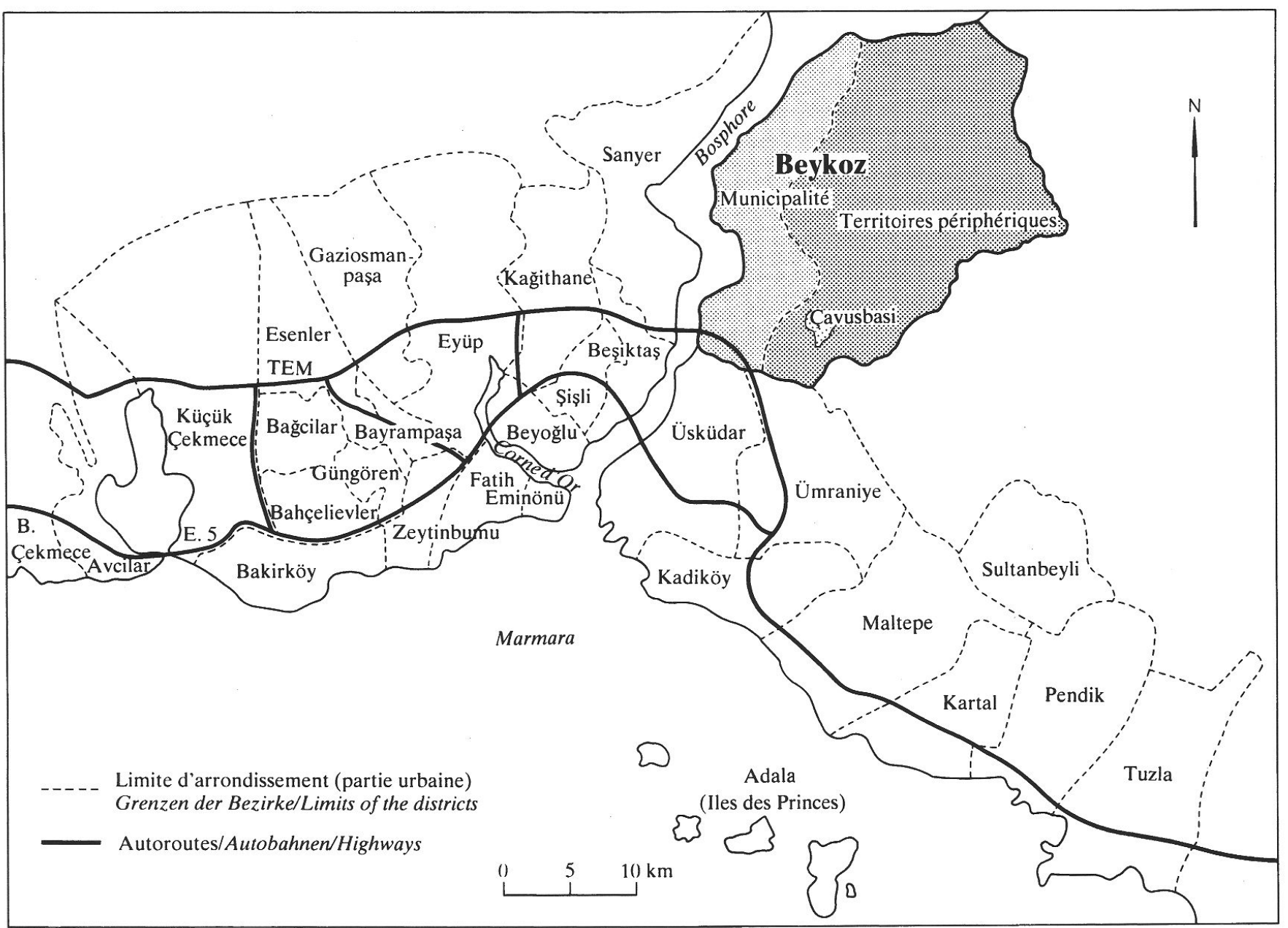

Fig. 1: L'arrondissement de Beykoz, dans l'aire métropolitaine d'Istanbul

The location of the Beykoz district within the agglomeration of Istanbul

Der Distrikt Beykoz im Verdichtungsraum Istanbul

Conception: J.-F. PÉRousE, réalisation: L. BAUMANN

\section{Méthode et sources}

L'analyse qui suit s'appuie sur une base de données des cités sécurisées d'Istanbul (plus de 430 ont été saisies de 1999 à début juin 2003), base élaborée à partir des publicités et des articles relatifs aux cités parus dans la presse turque comme dans les revues spécialisées dans l'immobilier. Quatre critères discriminants, discutables, ont été retenus pour circonscrire le phénomène complexe des cités sécurisées: la fermeture, et le dispositif de surveillance qui lui est associé, la présence d'équipements réservés, la gestion privée et le fait qu'il s'agit d'un ensemble de résidences principales. Cette base multi-critères vise à assurer un suivi du développement des cités à l'échelle de l'ensemble du département d'Istanbul et de leurs conditions de construction et de commercialisation. Par ailleurs, notre travail repose sur une série d'observa- tions directes in situ et d'entretiens qualitatifs effectués directement dans les cités ou aux abords de celles-ci, depuis 1998.

\section{Un arrondissement très opportun, sur une marge active de l'aire urbaine}

\subsection{Au moins une trentaine de cités terminées ou en passe de l'être}

A partir de nos données, on dénombre, en décembre 2002, 30 cités sécurisées. Comme le montre le tabl. 1, dans lequel nous ne faisons figurer que les cités identifiées de visu (soit 26 sur 30), ces cités sont de taille et de conformation très variables. Elles représentent, en décembre 2002, un parc de logements de plus de 6000 unités, dont la ville privée d'Acarkent concentre à elle seule plus de la moitié. 


\begin{tabular}{|c|c|c|c|}
\hline Nom & Lieu dit & Type de logement & Nombre de logements \\
\hline Acarkent & Forêt de Saip Molla & Villas et appartements & 3364 \\
\hline Beykoz Konaklari & Forêt de Saip Molla & Villas & 401 \\
\hline Hisar Evleri & Göksu & Appartements & 750 \\
\hline Göksu Evleri & Göksu & Appartements & 500 \\
\hline Özdoktorlar & Kavacik & Appartements & 382 \\
\hline Hekimler sitesi & Kanlica & Appartements & 300 \\
\hline Özhavacilar & Kavacik & Appartements & 300 \\
\hline AlYap 2 & Otag Tepe (Anadoluhisari) & Appartements & 220 \\
\hline Dogan Evler & Görele & Appartements & 88 \\
\hline Otag Evler & Otag Tepe (Anadoluhisari) & Appartements & 80 \\
\hline HBM International & Kanlica & Villas & 36 \\
\hline $\begin{array}{l}\text { Beykoz Günyüzü } \\
\text { Konaklari }\end{array}$ & Pasabahçe & Villas & 33 \\
\hline Otag Tepe Evleri & $\begin{array}{c}\text { Otag Tepe } \\
\text { (Anadoluhisari) }\end{array}$ & Villas & 30 \\
\hline Elmali Bahçe & Polonezköy & Villas & 30 \\
\hline Has Bahçe Evleri & Kandilli & Villas & 27 \\
\hline Hisartepe Evleri & Otag Tepe (Anadoluhisari) & Villas & 25 \\
\hline Hidiv Köskleri & Kanlica & Villas & 22 \\
\hline Pasabahçe Evleri & Pasabahçe & Appartements & 20 \\
\hline H.B. Villalari & Polonezköy & Villas & 16 \\
\hline Polonez Köskleri & Polonezköy & Villas & 15 \\
\hline Erkan Villalari & Göksu & Villas & 15 \\
\hline Maya Çubuklu & Kanlica & Villas & 13 \\
\hline Beykoz Koru Köskleri & Centre & Villas & 10 \\
\hline Mentese Evleri & Görele & Villas & 10 \\
\hline Polonez Villalar & Polonezköy & Villas & 6 \\
\hline $\begin{array}{c}\text { Bey Konaklari } \\
\text { Total }\end{array}$ & Küçüksu & Villas & $\begin{array}{c}6 \\
6699\end{array}$ \\
\hline
\end{tabular}

Tabl. 1: Les cités de Beykoz et leurs principales caractéristiques

Residential complexes in Beykoz and their main characteristics

Die Wohnkomplexe von Beykoz und ihre hauptsächlichen Merkmale

Du point de vue de la taille des cités, le tabl. 1 montre l'ampleur des écarts - de 6 à plus de 3000 logements -, qui rend l'objet «cités privés» extrêmement hétéroclite et oblige à une grande prudence dans l'analyse, pour éviter toute réduction et tentative de construction d'un objet d'étude cohérent. Il recouvre des réalités très différentes en termes de paysage produit et d'espace vécu.

Par ailleurs, ces cités sont loin d'être toutes également habitées. On peut même parler d'un taux d'occupation encore faible: d'après nos observations et entretiens, moins de la moitié du parc de logements est en fait occupé à la fin de l'année 2002; ce qui n'empêche pas que plus de la moitié soit déjà acheté. En 2000, la presse turque n'avait d'ailleurs cesse d'évoquer les efforts du patron d'Acarkent en vue de vendre les 40 villas, sur 1452 , alors non encore vendues, malgré les plans de paiement échelonné proposés sur dix ans).
Il y a donc une distorsion flagrante entre l'emprise spatiale, la visibilité de ces cités et leur population effective encore modeste. En ce sens, les cités sont une des expressions de l'étalement urbain en cours à Istanbul et d'un développement effréné de l'aire urbaine qui s'opère plus par l'offre que par la demande. On est donc en droit de se demander dans quelle mesure ce produit immobilier de haut de gamme - en tout cas dans ses prétentions -, reproduit à l'envi en fonction des opportunités, sans étude de marché sérieuse, pourra trouver une clientèle. L'intense mouvement de revente,l'apparente stagnation de certaines opérations et la dégradation de nombreux bâtiments en attente sont autant d'indices militant dans ce sens. L'interruption de certains chantiers est aussi due aux difficultés inhérentes à des opérations qui fonctionnent en autofinancement, à la crise bancaire de 2000-2001, voire à des blocages juridiques liés au caractère illégal de plusieurs cités, au regard du statut du sol. 
En ce qui concerne les formes de logement, on constate que plus de la moitié du parc de logements est constitué de logements en immeubles. Les 400 appartements des Hauts du Bosphore - répartis en 5 blocs comprenant 80 unités chacun -, au sein de la ville privée d'Acarkent, d'abord réputée pour ses villas, sont révélateurs de l'extrême diversité de l'offre immobilière dans ces cités, dans la mesure où l'on oscille entre les studios (1 512 proposés au total; au nom éloquent d' «Espaces de l'amour», ces studios - de 69 à $122 \mathrm{~m}^{2}$ -, sans vue sur le Bosphore, sont situés près du Centre de Sports et de Loisirs d'Acarkent, le Coliseum) et l'énorme villa de plus de $780 \mathrm{~m}^{2}$ habitables, en passant par l'appartement de $250 \mathrm{~m}^{2}$ dans les Hauts du Bosphore. Cette diversité, au sein d'une même opération, et les infléchissements survenus au fil du temps dans le programme de construction, traduisent la volonté des promoteurs de toucher des publics cibles plus larges et la difficulté de commercialiser une forme unique de produit immobilier. En effet, au moment du lancement du projet à la fin des années 1980, il n'était pas question de construire des appartements; Acarkent se voulait tout simplement la plus grande ville de villas de Turquie. Par rapport à Acarkent, la cité de Beykoz Konutlari apparaît plus homogène, dans la mesure où elle ne compte que des villas, au nombre de 401, de type assez proche.

\subsection{Une série d'atouts, qu'ils soient «donnés» ou construits}

Un cadre naturel recherché et un désir d'isolement. Les promoteurs de cité ont recherché en priorité pour leurs clients des terrains situés soit à proximité du Bosphore, soit sur les hauteurs avec vue sur le Bosphore, soit des terrains au cœur des forêts qui subsistent. De ce fait, l'argumentaire publicitaire joue invariablement sur ces qualités de l'environnement, opposées aux miasmes qui rendent le centre d'Istanbul invivable. Selon le marketing pratiqué, il s'agit donc de fuir les arrondissements centraux délétères (et diabolisés), pour se réfugier dans des niches protégées et réservées.

En outre, quand l'environnement plaisant n'est pas présent, il est construit, à grands renforts d'aménagements et de mises en paysage. En conséquence, une des caractéristiques des cités, pas seulement à Beykoz, réside dans le contraste stupéfiant entre le soin apporté aux espaces à l'intérieur des cités et le négligé des environs immédiats, situés hors de la souveraineté des dites cités. Ce divorce illustre de façon saisissante l'absence complète d'articulation entre initiative privée, ponctuelle, et gestion publique de l'espace banal environnant par des collectivités locales sans ressource.

L'isolement, enfin, participe des atouts les mieux mis en valeur, dans la mesure où il concrétise le souci de distinction - le terme étant entendu à la fois dans sa dimension spatiale et sociale - qui semble animer les acheteurs de logements en cités sécurisées. Il est aussi concrétisé par les enceintes érigées autour de chaque cité, de plus en plus doublées d'enceintes végétales pour accentuer l'effet d'isolement. Pour autant, et c'est l'un des arguments clés des promoteurs, les cités ont la prétention d'être à la fois loin et proche d'Istanbul, la métropole étant considérée tantôt comme un nœud de facteurs négatifs à fuir, tantôt comme une ensemble irremplaçable de ressources.

Un sous-sol réputé plus sûr face aux risques sismiques. Il est par ailleurs indéniable que depuis les séismes de 1999, Beykoz bénéficie d'une bonne réputation, compte tenu des avantages relatifs de son soussol, que les vendeurs de cités privées ne manquent pas de mettre en avant dans leurs discours promotionnels. Sans entrer dans les détails géologiques et lithologiques, les études du sous-sol conduites ces dernières années pour circonscrire précisément les zones à risques dans l'aire urbaine, font en effet apparaître Beykoz comme un havre de sûreté relative. A Acarkent, tout acheteur potentiel se rendant dans le showroom se voit remettre avec les brochures de luxe présentant la ville privée, une copie du rapport scientifique commandité par le constructeur en septembre 1999, et signé par des spécialistes de l'Université d'Istanbul, assurant que le sous-sol d'Acarkent est le plus sûr de tout Istanbul.

De rapides liaisons routières. $\mathrm{La}$ construction du deuxième pont sur le Bosphore, achevé en 1989, a inauguré pour Beykoz une ère nouvelle, en accélérant de façon spectaculaire le processus d'urbanisation de fait. En effet, à partir de ce moment, les conditions pour une intégration de Beykoz à l'aire urbaine ont été assurées. Dès lors, il n'est pas étonnant de constater que toutes les publicités pour les cités de Beykoz mettent en avant la proximité du deuxième pont et des accès au second périphérique dit Trans European Motorway (TEM), ainsi que la qualité de la desserte, quitte à contracter l'espace-temps, en ne tenant pas compte des possibles encombrements à certaines heures. De même, on constate que l'ouverture de l'autoroute d'Elmali a eu un effet stimulant sur les projets de construction, cette voie rapide à travers les forêts de Beykoz faisant figure de pénétrante ouvrant de nombreuses opportunités aux constructeurs. Dans ce cas, il est difficile de ne pas faire le lien entre les projets d'infrastructures routières et les projets des constructeurs de cités; en d'autres termes, les connivences entre les constructeurs et certains membres de l'administration semblent évidentes. Cette autouroute, en fait une voie rapide 2 fois 2 voies, permet de se rendre de Kavacik aux Beykoz Konaklari et à Acarkent en moins de 5 minutes. Pour les Beykoz Günyüzü, dont 


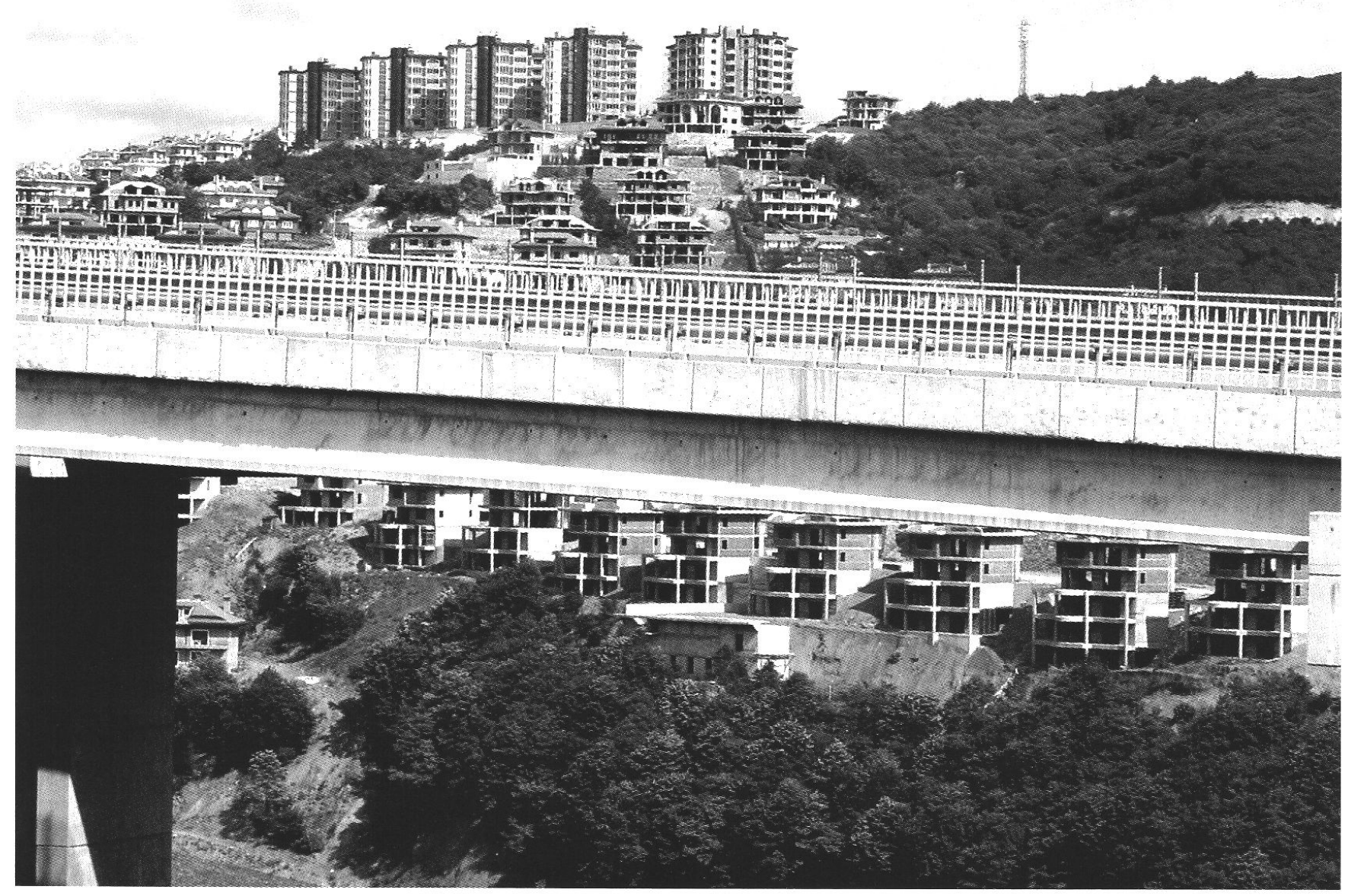

Photo 1: Les cités sécurisées d'Acarkent et de Beykoz Konaklari, avec le viaduc nouvellement construit, en juillet 2002

The gated communities Acarkent and Beykoz Konaklari, with the newly built viaduct, in July 2002

Die bewachten Wohnkomplexe Acarkent und Beykoz. Konaklari, mit einem neu errichteten Viadukt, im Juli 2002

Photo: J.-F. PÉrouse

la construction a commencé au début de l'année 2001, il y a exacte coïncidence spatiale et temporelle entre le chantier de la cité et celui de la voie express. Certaines villas sont même construites sous le viaduc en voie d'achèvement (photo 1). Cependant, on ne peut s'empêcher de s'interroger sur les effets négatifs - en termes de nuisances sonores notamment - de cet axe routier rapide, tant il passe près des Beykoz Konaklari. L'ouverture de nouveaux axes routiers contribue à la dégradation de l'environnement des cités sécurisées et donc à leur durabilité douteuse.

Le nouveau pôle tertiaire de Kavacik. Suite à l'ouverture du deuxième pont, le secteur de Kavacik, en situation de tête de pont sur le second périphérique, a vu la construction rapide d'immeubles de bureaux, au point de faire désormais de ce quartier un des principaux pôles tertiaires supérieurs de l'aire urbaine. En conséquence, les vendeurs de cités sécurisées, ne manquent pas de faire valoir cette proximité de Kavacik, possible pôle d'emplois pour la clientèle ciblée.

\subsection{Des infractions multiples dans des zones de quasi non-droit}

Un arrondissement sans contrainte? En droit, plus de $90 \%$ de la superficie de l'arrondissement (BASTAR 2000) est non constructible ou à des conditions extrêmement strictes, puisque dépourvue de plan d'aménagement au 1/5 000ème élaboré par les pouvoirs locaux, en conformité avec le plan d'agglomération au 1/25 000ème (avec ratification par la mairie centrale). En outre, la quasi-totalité de l'arrondissement est classée zone 
protégée (Sit alani), du fait de la double présence du Bosphore et des forêts. Par conséquent, toute construction (et même toute modification du bâti existant) devrait être soumise à l'autorisation préalable du Conseil pour la Protection des Biens Culturels et Naturels (MutLu 2001). Pourtant, un régime d'autorisation provisoire existe, en attendant que l'arrondissement soit doté d'un réel document d'urbanisme; ce qui crée des conditions favorables à de nombreux abus et appropriations indues. En d'autres termes, l'immensité peu contrôlable de l'arrondissement, conjuguée aux vides juridiques persistants, font de ce territoire un complexe d'opportunités pour les constructeurs sans scrupule.

Les infractions aux lois sur la forêt. Les cités sécurisées illustrent le caractère très diversifié, socialement parlant, du phénomène de la construction illégale à Istanbul, qu'on aurait tort de réduire à l'auto-construction par des ménages démunis. En d'autres termes, l'illégal peut avoir un visage très «haut standing». Beykoz offre, à cet égard, des exemples flagrants d'entorses au droit sur les forêts, sur un mode luxueux. Selon SANER (2000: 209), sur un total de 12070 hectares extraits du statut de forêt ces dernières années sur la rive asiatique du département d'Istanbul, plus de 13\% l'ont été dans le seul arrondissement de Beykoz (soit 1650 hectares). Si le Droit de la Forêt turc autorise des personnes et des institutions privées à faire l'acquisition de terrains, celle-ci doit se faire dans des conditions précises et strictement réglementées. Ainsi, seuls $6 \%$ de la surface des terrains de forêt acquis peuvent, selon la loi, être urbanisés (il s'agit là d'une sorte de coefficient d'occupation du sol). Deux cas d'acquisitions récentes de terrains forestiers à Beykoz, pour construire des cités sécurisées, dont la plus grosse de Turquie (Acarkent), prouvent combien les textes de loi peuvent être contournés. Il s'agit des forêts dites de Saip Molla. La première était située à Çavusbasi; sur les 229 hectares acquis, $68 \%$ - dont $32 \%$ pour l'habitat à proprement parler - ont été accaparés et transformés (pour construire 1742 villas et leurs équipements). La seconde forêt de Saip Molla, d'une superficie de 96 hectares, a connu les mêmes entorses au règlement, avec la construction de 401 villas (Beykoz konaklari) et de leurs équipements annexes. Dans les deux cas, sans des complicités dans l'appareil d'Etat et dans les administrations locales, cette appropriation massive, au mépris de la loi, n'aurait évidemment pas pu avoir lieu.

\subsection{Continuités: des yali aux néo-kösk}

Depuis l'époque ottomane au moins, Beykoz est réputé parmi les classes dirigeantes pour ses agréments. En bord du Bosphore, comme au cœur de la forêt, les notables ottomans ont fait construire à partir du XVII e siècle, qui un yali (résidence de standing en bois, édifiée sur les rives du Bosphore), qui un kasir (sorte de castelet) sur les hauteurs dominant le Bosphore, qui un kosk (pour la chasse ou le repos), dans les bois. Le castel du dernier khédive d'Egypte, Abbas Hilmi, édifié au début du XXe siècle, est un des derniers indices de cette prédilection ottomane. Ce n'est qu'après l'ouverture du second pont, déjà évoquée, que Beykoz redevient un territoire de prédilection, selon de nouvelles modalités et de façon plus massive. Le démarrage du chantier des cités Özhavacilar et Özdoktorlar, en 1991, nous semble constituer une bonne date de référence, indice du début du développement des cités sécurisées dans l'arrondissement, pour la résidence permanente cette fois-ci. On peut ajouter à cette chronologie sommaire les tremblements de terre de l'année 1999, qui ont eu pour effet, compte tenu de la solidité supposée du sous-sol de Beykoz, d'accroître encore l'attractivité de l'arrondissement, auprès des classes aisées en tout cas. Enfin, compte tenu du potentiel en espace et en opportunités qu'offre Beykoz, on comprendra aisément que le mouvement de construction est loin d'être terminé. Par conséquent, l'histoire des cités rivées à Beykoz, contrairement à d'autres arrondissements où, du fait de la densité bâtie, les perspectives sont limitées - si ce n'est par fermeture du bâti existant ou par apparition de cités verticales -, est loin d'être close.

Nous avons donc affaire à un processus récent, qui fait rejouer des formes passées de distinction sociale. A cet égard, la reprise des noms jadis utilisés pour désigner l'habitat des élites ottomanes (konak, kösk) nous paraît être révélatrice d'un souci d'établir une continuité entre ces modes sélectifs de résidence.

\section{Bref essai de typologie: des degrés certains dans la distinction}

Pour une première typologie on peut sélectionner quatre critères principaux - nature du promoteur, gigantisme, standing/profil de la population résidente et localisation -, qui nous semblent, en l'état des recherches, suffisamment discriminatoires pour pouvoir à la fois différencier et caractériser globalement les cités.

\subsection{Petits groupes, grands groupes de construction et coopératives}

Une partie des cités a été construite à l'initiative de grands groupes, dont plusieurs sont spécialisés dans la construction et disposent parfois même d'une envergure internationale. Le groupe Tepe Insaat, bâtisseur des Beykoz Konaklari, est l'archétype de ces groupes. Il est connu pour avoir mené à bien des chantiers dans le monde arabe et dans le monde russe, où il a accumulé une expérience qui fait sa réputation. C'est un des grands groupes turcs de la construction, à qui ont 


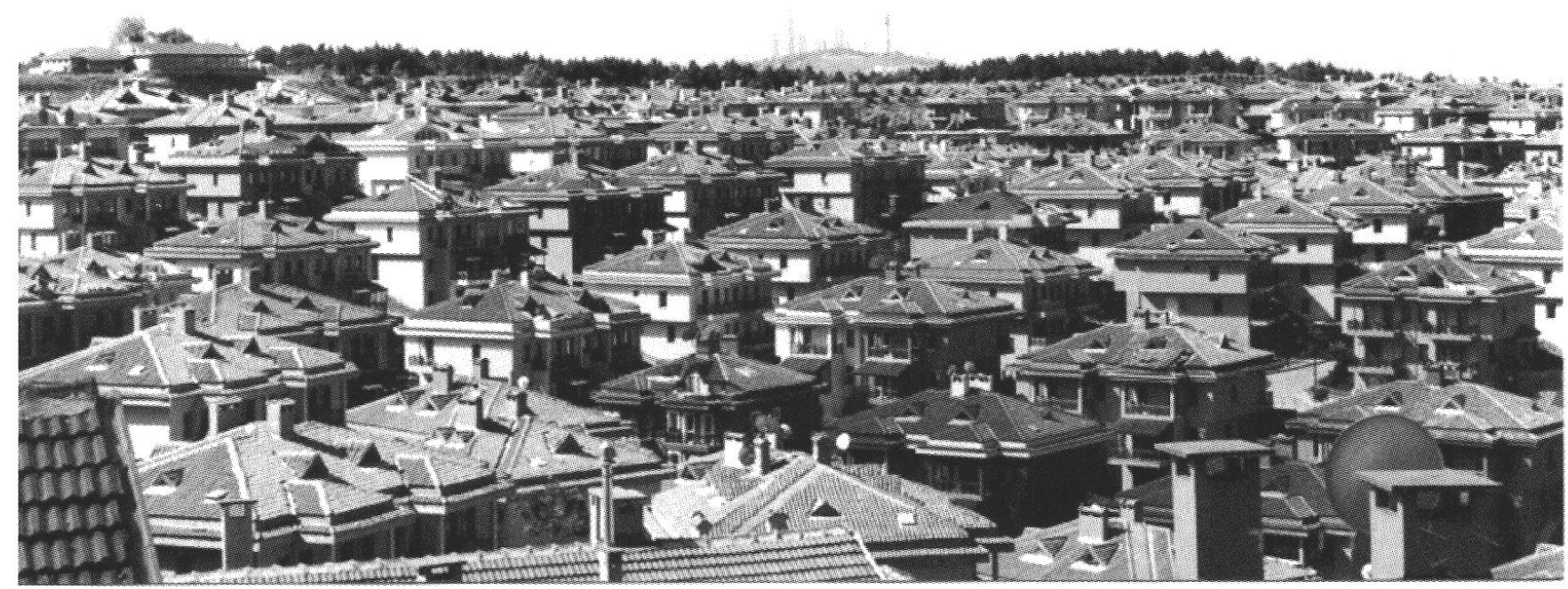

Photo 2: Densité du bâti à Göksu Evleri, en juillet 2002

Building density in Göksu Evleri, July 2002

Dichte der Bebauung in Göksu Evleri, Juli 2002

Photo: J.-F. PÉrouse

été confiées des opérations d'importance nationale comme l'extension de l'aéroport d'Istanbul -, qui peut profiter de son assise pour imposer certains de ses projets aux marges de la légalité locale. Le groupe Acarlar Sirketler - constructeur d'Acarkent - relève aussi de cette catégorie de grand constructeur, sans pour autant disposer d'une réputation internationale: il s'agit plutôt d'un groupe disposant de solides relations dans l'appareil d'Etat et administratif, sans lesquelles il n'aurait pas acquis son envergure actuelle.

Les petits groupes représentent une catégorie d'acteurs spécifique, dans la mesure où il s'agit souvent de sous-traitants de grands groupes de construction, qui, pour une opération limitée, deviennent à leur tour promoteurs à part entière. Ces petits groupes locaux profitent de l'expérience et des relations accumulées au cours du grand chantier auquel ils ont été associés, pour réaliser leur propre opération, souvent sur un mode plus modeste. Toutes les petites cités situées dans l'orbite d'Acarkent et des Beykoz Konaklari, comme Dogan Evleri ou Mentese Evleri, relèvent de cette logique périphérique ou d'induction.

Il convient enfin de mettre à part les coopératives, dans la mesure où elles correspondent à un mode d'organisation original, dont la construction de cités traduit les dérives spéculatives actuelles. Conçues dans les années trente sur une base corporative, sociale et non lucrative, les coopératives se sont au fil du temps détournées de leurs objectifs premiers, pour investir le marché spéculatif de la construction, en continuant néanmoins à profiter des avantages traditionnellement consentis par l'Etat (accès au terrain et financement). La «Cité des Médecins» (Hekimler sitesi) une des premières cités de Beykoz -, celle des «Docteurs» (Özdoktorlar sitesi) ou celle des «Aviateurs» (Özhavacilar sitesi) constituent des exemples typiques de cette catégorie. En effet, toutes ces cités-coopératives se caractérisent par une dérive spéculative, par une recherche de standing distinctif et par un processus de franche fermeture qui les distinguent en dernière analyse peu des autres cités, si ce n'est dans le recrutement de leur population.

\subsection{Cités géantes (villes) et micro-cités}

Comme le suggère le tabl. 1 , entre des cités de plusieurs centaines, voire milliers, de villas ou de logements et des cités de quelques villas, la différence est notable. Il est donc possible de distinguer les cités, en termes de visibilité dans le paysage, comme en termes d'emprise spatiale. Pour autant, il n'existe pas de liens directs entre la taille et le standing. On trouve des cités très sélect de petite taille (comme les Otag Tepe Evleri ou les Hisartepe Evleri) et de grande taille. Mais certaines grandes cités, comme les cités coopératives le long du Göksu (Özhavacilar et Özdoktorlar) n'ont pas un recrutement trop sélectif.

Lié à ce critère de la taille, on pourrait ajouter celui des partis pris urbanistiques et architecturaux. De fait, les cités se distinguent par des choix de concep- 


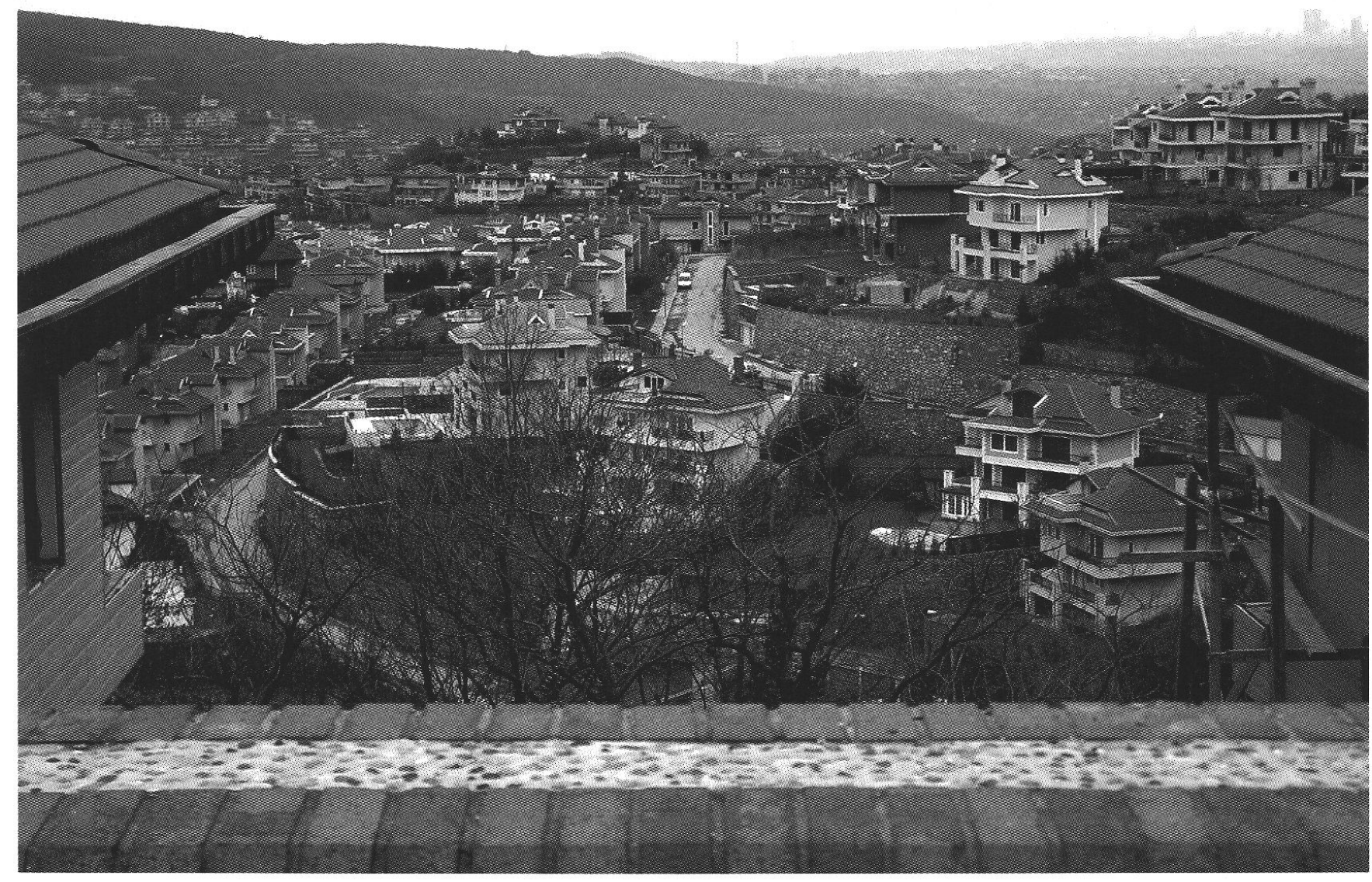

Photo 3: Densité du bâti à Acarkent, en décembre 1999 Building density in Acarkent, December 1999

Dichte der Bebauung in Acarkent, Dezember 1999

Photo: J.-F. PÉrouse

tion extrêmement divers. Aux formes néo-ottomanes - ou prétendument telles, mais les noms utilisés constituent de bons indices -, s'opposent des choix architecturaux empruntant à des références européennes ou plus fréquemment américaines. A cet égard, les Beykoz Konaklari se distinguent: un ancien bâtiment - un kiosque de chasse édifié en 1904 - y a été promu en cœur de la cité, auquel les formes architecturales des villas s'efforcent de faire écho. De même, la densité de l'occupation du sol, qui n'est pas - on l'a vu conforme aux coefficients officiels, est un bon critère de différenciation. A ce titre, sans parler évidemment des cités d'immeubles, les Göksu Evleri de Kavacik, avec moins de $400 \mathrm{~m}^{2}$ par villa, constituent un exemple limite, tant la densité du bâti y est forte (photo 2). Dans une moindre mesure, la surface par villa à Acarkent - $1500 \mathrm{~m}^{2}$ (photo 3 ) - est bien inférieure à celle des Beykoz Konaklari.

\subsection{Une sélection plus ou moins poussée}

Par ailleurs, les cités ont un caractère plus ou moins «chic». Cela se mesure au profil de la population, c'està-dire au type d'emploi des habitants et au niveau de revenus, à la qualité et à la diversité des services proposés, ainsi qu'aux prix pratiqués, à la vente ou plus rarement à la location. L'échelle des prix est, quant à elle, éloquente, puisqu'elle varie dans une fourchette allant de 150000 US dollars à 2,5 millions, pour des produits presque finis, décoration et ameublement non compris.

En ce qui concerne le profil socio-professionnel, on peut opposer les cités habitées par de grands industriels et hommes d'affaires souvent soucieux de discrétion - cités de taille modeste, comme Hisartepe et Otag Tepe Evler -, aux cités habitées par des personnes enrichies rapidement, qui travaillent dans le secteur des 
médias, du showbusiness, de la publicité ou du sport professionnel. On dispose, de ce point de vue, d'un document intéressant - à lire avec précaution cependant, dans la mesure où les pratiques de dissimulation sont fréquentes - constitué par la liste des premiers acheteurs d'Acarkent en 1988 («ville» qui ne portait pas encore ce nom, mais celui d'Uyumkent, «Ville Harmonie»). Parmi ces résidents, on remarque des membres de la haute administration et du monde politique national (dont un futur ministre des forêts, HASAN EKINCI) et local, des représentants célèbres du secteur immobilier turc et de grands commerçants, parfois figures éminentes de la communauté juive d'Istanbul. Mais par rapport au profil des premiers habitants, il semble que la population se soit diversifiée pour accueillir davantage d'enrichis très récents, et d'étrangers, souvent russes. Par exemple, on sait que plusieurs mannequins turques connues habitent à Acarkent, dont elles apprécient le centre sportif Coliseum qui fait désormais partie de la réputation de cette cité (c'est même une vitrine, destinée à séduire les non résidents aisés qui y viennent). De même, plusieurs personnalités du monde des médias et du sport professionnel habitent maintenant à Acarkent (comme à Beykoz Konaklari, qui affiche pourtant des prétentions culturelles plus nettes). Dans certaines cités, les futurs habitants ont été triés sur le volet, avec un droit de regard exercé par les plus anciens, en fonction de critères non pas seulement financiers, mais plutôt culturels (niveau d'éducation, type de diplôme). On y prétend en tout cas qu'un public cible précis y a été défini. Mais au fil du temps, par le jeu de la revente que ne maîtrise pas le promoteur/développeur, le contenu social d'une cité peut se modifier, de la même façon que son environnement peut rapidement se dégrader.

En ce qui concerne les équipements et services proposés (qui ne sont pas tous offerts, loin de là), la différenciation s'opère à la fois en termes de qualité et de diversité de l'offre, à partir d'un concept unique, celui de la garantie d'une vie sûre, confortable et quasi autarcique. Les équipements scolaires et sanitaires paraissent les plus distinctifs. Ainsi, l'implantation d'un établissement scolaire privé, aussi prestigieux que le Ted Istanbul Koleji, à proximité d'Acarkent et des Beykoz Konaklari, ne relève-t-elle pas du hasard et ne signe-t-il pas le type de population habitant dans ces cités?

\subsection{Trois types de localisation}

Les collines sur le Bosphore. Les premières cités de Beykoz (Hekimler), dans la continuité des implantations ottomanes, sont situées sur les hauteurs dominant le Bosphore. Ici, c'est la vue, l'aération et la discrétion - compte tenu de la végétation entourant ces cités - qui l'emportent. La majorité des cités se range dans cette catégorie, avec des degrés quant à la qualité de la vue. Si la cité du Göksu fait toute sa publicité sur la proximité du Bosphore, seul un nombre réduit de villas ont en fait une vue sur celui-ci. Et quand on s'attache aux prix pratiqués au sein de la cité, des écarts importants apparaissent en fonction de la vue, cela allant du simple au double. Plusieurs pôles se détachent: celui de Kavacik-Göksu, pour les cités coopératives à densité élevée, ceux d'Otag Tepe et de Kanlica, avec des cités de taille plus réduite et de très haut standing Fig. 2).

Le cas de Çavusbasi: la soudaine naissance d'une ville privée. Ici, la proximité du Bosphore, offrant quelques belles perspectives des hauteurs - comme pour les 5 blocs d'appartements d'Acarkent - se combine avec l'environnement forestier de plus en plus malmené. Le contexte juridique flou paraît avoir été déterminant dans le développement de ces cités. En effet, avant avril 1999, date à laquelle Çavusbasi est devenu une municipalité de second rang (belde belediyesi) à part entière, le développement urbain s'est fait sans aucun contrôle, même aux abords du barrage-réservoir d'Elmali, pourtant censé être protégé; les quelques destructions de villas de standing, ordonnées par l'Administration des Eaux en 1996, n'ont eu qu'une portée momentanée. Selon les photographies aériennes, entre 1995 et 1999, plus de 3500 constructions illégales ont été réalisées à Çavusbasi (qui compte aujourd'hui 9 000 bâtiments en tout), dont la ville privée Acarkent, qui s'étend sur des dizaines d'hectares. De la sorte, la population de la bourgade a ainsi été multipliée par plus de 5 entre 1990 et 2000, pour dépasser 20000 en 2002. Petite enclave de quelques $\mathrm{km}^{2}$, au sein des territoires périphériques, Çavusbasi dispose maintenant d'une relative autonomie dans la définition de sa politique de construction, tout en étant obligée de respecter des règles strictes de faible densité. Mais entre la municipalité de la bourgade et l'administration de la ville privée d'Acarkent, le jeu apparaît inégal.

Les forêts et les villages périphériques: des réserves ponctuellement investies. Hors des limites de la municipalité de Beykoz et de celles de Çavusbasi, dans des territoires sous contrôle de l'administration préfectorale ou de l'administration des forêts, le développement des cités s'opère sur un mode extensif et sauvage. Parmi ces cités, qui privilégient l'éloignement par rapport à la foule stambouliote et l'intimité jalouse avec la forêt, celles de Polonezköy sont les plus révélatrices. Ce village fréquenté le week-end par les Stambouliotes aisés, notamment dans le cadre de country clubs (le Polonez Country Club, sans doute le premier, a été ouvert en 1981) et d'hôtels, est depuis quelques années habité de façon plus assidue, dans le cadre de quelques cités peu denses, édifiées en dépit des strictes interdictions qui protègent ce village célèbre. Les formes d'habitation y demeurent diverses, mais le mode permanent 


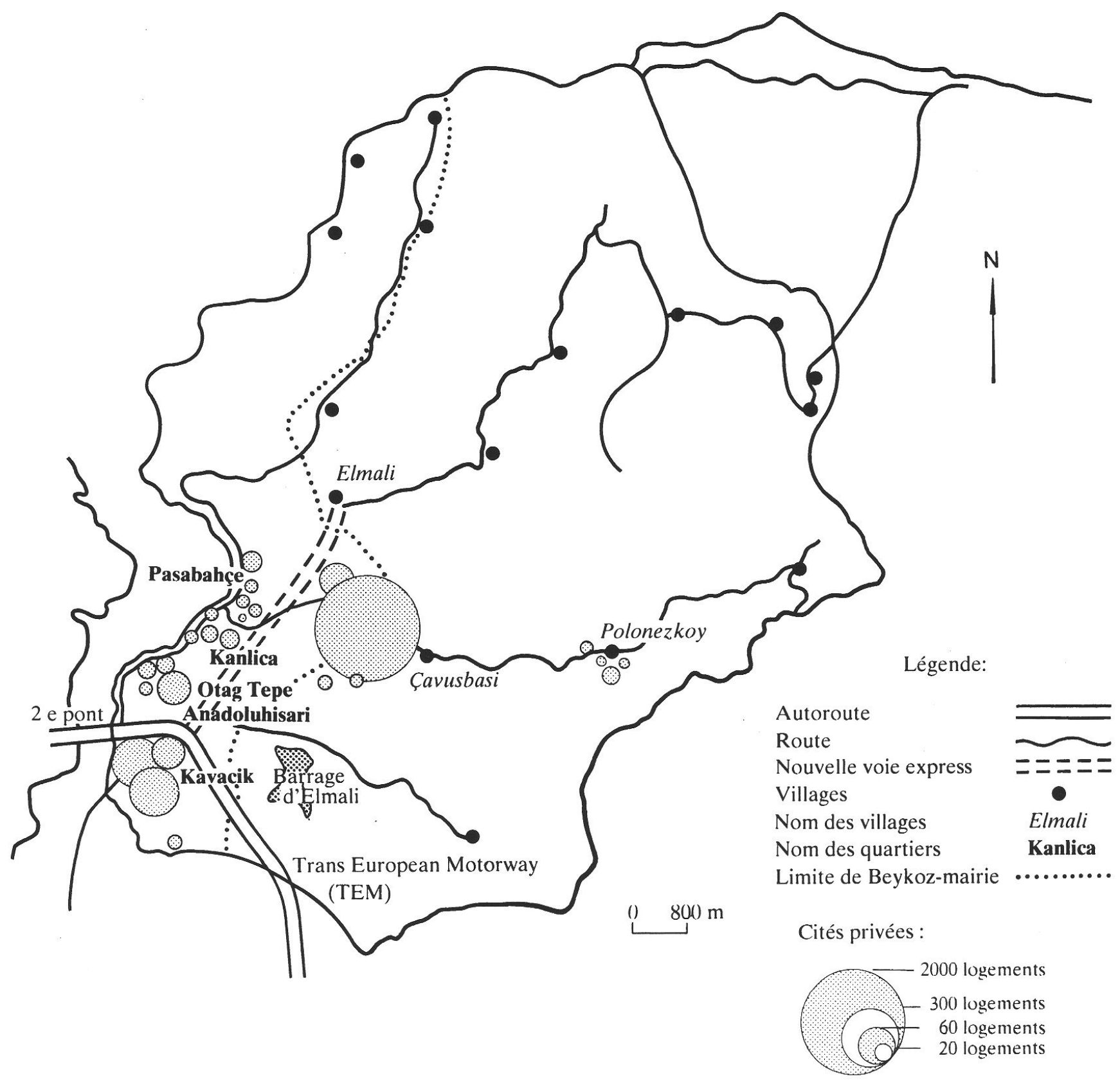

Fig. 2: Principales localisations et concentrations de cités sécurisées

Main locations and concentrations of gated communities

Wichtigste Standorte und Konzentrationen von bewachten Wohnkomplexen

Conception: J.-F. PÉRouse; réalisation: L. BAUMANN

tend à s'imposer, avec un souci d'investir une histoire locale non turque (ANTONOWicz-BAUER 1990), érigée en ressource du marketing territorial (fig. 2).

\section{Résultats}

1. Il n'existe pas un type dominant de cité sécurisée. D'où la nécessité de dégager des critères distinctifs, à la fois stricts et souples, pour éviter une disso- lution de l'objet d'étude, sachant que le phénomène s'inscrit dans des tendances plus vastes, qu'il concentre et exacerbe en quelque sorte.

2. Le rôle primordial des constructeurs-développeurs, partie prenante d'un système politique local et interdépendant du fait de relations de sous-traitance ou de co-traitance, incite à parler d'une émergence par l'offre de construction à destination d'une clientèle encore vague. 
3. Seule une étude développée dans le temps permet de prendre en compte les dérapages et de formuler l'hypothèse que l'on a affaire à des produits immobiliers à courte durée de vie, ayant cependant la prétention de s'inscrire dans la continuité de formes résidentielles passées, mais distinctives.

\section{Références bibliographiques}

Амaто, P.W. (1970): Elitism and Settlement Patterns in the Latin American city. - In: Journal of the American Institute of Planners 36: 96-105.

Antonowicz-BAUER, L. (1990): Polonezköyü-Adampol. Istanbul: Çelik Gülersoy Vakfi, Istanbul Kütüphanesi, N.3.

BALI, R.N. (1999): Loin de la folle populace. - In: Birikim 123, juillet 1999: 35-46 (en turc).

BARTU, A. (2002): Le «quartier» comme concept excluant.- In: Istanbul 40: 84-86 (en turc).

BASTAR, S. (2000): Je suis pieds et main liés (en turc). In : Sabah-Istanbul, quotidien, 11.07.20, Istanbul: 1.

Blakely, E.J. \& M.G. SNYDER (1997): Fortress America. Gated Communities in the United States. - Washington D.C., Cambridge/Mass.: Brookings Institution Press.

CoY, M. \& M. PöHLER (2002): Condomínios fechados und die Fragmentierung der brasilianischen Stadt. Typen - Akteure - Folgewirkungen. - In: Geographica Helvetica 4: 264-277.

Davis, M. (1990): City of Quartz: Excavating the future in Los Angeles. - London: Verso.

Fischer, K. \& C. PARnReiter (2002):Transformation und neue Formen der Segregation in den Städten Lateinamerikas. - In: Geographica Helvetica 4: 245-252.

Mutuu, N. (2001): Contre Goliathe. - In: Time Out Istanbul, février 2001, N.102, Istanbul: 66 (en turc).

ÖNCÜ, A. (1999): La mythologie de la «maison de vos rêves» a atteint Istanbul en dépassant les frontières culturelles. - In: Birikim 123, juillet 1999: 26-34 (en turc). SANER, A.Ü. (2000): Devletin Ranti Deniz (la mer, rente d'Etat). - 3ième édition, Istanbul: Iletisim.

Sites Internet de quelques cités:

Acarkent: http://www.acarkent.net http://et

www.coliseumcenter.com

Beykoz Konaklari: http://www.beykozkonaklari.com

\section{Résumé: La sournoise émergence des cités dites sécurisées en Turquie. Le cas de l'arrondissement de Beykoz (Istanbul)}

Beykoz, arrondissement de la périphérie nord-est d'Istanbul, à proximité du Bosphore et de la mer Noire, connaît depuis une dizaine d'années des transformations importantes dues, notamment, au développement de cités sécurisées dans des sites choisis. Ces cités, dont deux peuvent être considérées du fait de leur taille comme de véritables «villes privées», traduisent, sous des formes éminemment variables, l'émiettement croissant de l'aire urbaine et la démission des pouvoirs publics.

Summary: The Hidden Emergence of Secured Housing Complexes in Turkey. A case study of the Beykoz district (Istanbul)

Located in Istanbul's north-western outskirts, near the Bosphorus and the Black Sea, the district of Beykoz has undergone radical change over the last few years due to the development of gated communities. Because of their size, two of these secured housing developments may be described as veritable «private cities». In addition, the phenomenon of secured housing complexes may be seen as a symptom of both the uncontrolled spread of the urban area and the general crisis of public space administration.

\section{Zusammenfassung: Die versteckte Entstehung bewachter Wohnkomplexe in der Türkei. \\ Das Beispiel des Beykoz-Bezirkes (Istanbul)}

Der Bezirk Beykoz, der in der nord-westlichen Peripherie von Istanbul in der Nähe des Bosporus und des Schwarzen Meeres liegt, hat in den letzten Jahren mit der Entwicklung von gated communities an ausgewählten Standorten eine grundlegende Transformation erfahren. Angesichts ihrer Größe können zwei dieser Siedlungen als veritable «Privatstädte» bezeichnet werden. Darüber hinaus kann das Phänomen betrachtet werden als Symptom eines unkontrollierten Wachstums der Siedlungsfläche und eines Rückzugs der öffentlichen Hand.

\section{Orientations didactiques}

- Quels types de sites privilégient les cités privées de cette périphérie d'Istanbul?

- Quel lien établir entre le développement du réseau routier et celui des cités?

- Quels sont les arguments spécifiques de vente en ce qui concerne la Cité à Beykoz et quels sont les problèmes soulevés en relation avec la construction de celle-ci?

- A quels profils socio-professionnels correspond le public visé par ces nouveaux produits immobiliers?

- Quelle articulation entre les cités et leur environnement?

Dr. Jean-François Pérouse, Institut Français d'Etudes Anatoliennes, Nuru Ziya Sokak, 22-P.K., 54-80072 Beyoglu, Istanbul, Türkei.

e-mail: jean-francois.perouse@ifea-istanbul.net

\section{Manuskripteingang/received/manuscrit entré le} 24.8.2003

Annahme zum Druck/accepted for publication/accepté pour l'impression: 8.12.2003 